\section{Incidence of inflammatory bowel disease by race and ethnicity in a population-based inception cohort from 1970 through 2010}

\author{
Satimai Aniwan (D, W. Scott Harmsen, William J. Tremaine and Edward V. Loftus, Jr.
}

\begin{abstract}
Background: Although inflammatory bowel disease (IBD) has been more predominant in white populations, an increasing incidence of IBD in nonwhites has been reported. We sought to evaluate the incidence rates and temporal trends of IBD by race and ethnicity.

Methods: The resources of the Rochester Epidemiologic Project were used to identify 814 county residents newly diagnosed with IBD from 1970 through 2010. Race was categorized into whites and nonwhites. Ethnicity was categorized into Hispanic and non-Hispanic. Incidence rates were estimated and adjusted for age and sex to the 2010 United States (US) population. Trends in incidence rates were evaluated by Poisson regression.

Results: The adjusted annual incidence rate of IBD for whites was 21.6 cases per 100,000 person-years [95\% confidence interval (CI), 20.0-23.1] and for nonwhites it was 13 per 100,000 (95\% Cl, 8.3-17.5). The incidence rates for whites and nonwhites increased by $39 \%$ and $134 \%$, respectively, from 1970 through 2010. The adjusted annual incidence rate of IBD for Hispanics was 15 cases per 100,000 person-years $(95 \% \mathrm{Cl}, 6.3-23.6)$ and for non-Hispanics was 20 per 100,000 (95\% Cl, 18.5-21.6). The incidence rate for Hispanics decreased by $56 \%$, while the rate for non-Hispanics increased by 33\%, from 1985 through 2010. In a Poisson regression, white race $(p<0.0001)$, a later year of diagnosis $(p<0.001)$, male sex $(p<0.001)$ and younger age $(p=0.009)$ were significantly associated with a higher incidence rate of IBD.
\end{abstract}

Conclusions: There were significant racial and ethnic differences in the incidence and temporal trends of IBD over the last four decades in this US population-based cohort.

Keywords: epidemiology, ethnicity, inflammatory bowel disease, race

Received: 1 August 2018; revised manuscript accepted: 4 January 2019.

\section{Introduction}

The inflammatory bowel diseases (IBD), consisting of Crohn's disease (CD) and ulcerative colitis (UC), are chronic inflammatory diseases and are commonly diagnosed at younger ages with relatively low mortality. ${ }^{1}$ The incidence of IBD continues to increase steadily in western countries, and the incidence of IBD in other continents has been rapidly increasing. ${ }^{2}$ Consequently, the global prevalence of IBD appears to be climbing and this will result in an increasing burden of IBD throughout the world in the future. ${ }^{3}$

Historically, IBD has been more predominant in white populations. Recently, the descriptive epidemiology of IBD in Olmsted County, MN, USA was updated through 2010. The incidence of CD increased from 6.9 cases per 100,000 person-years between 1970 and 1979 to 10.7 per 100,000 between 2000 and 2010, and the incidence of UC increased from 9.2 per 100,000 between 1970 and 1979 to 12.2 per 100,000 between 2000 and $2010 .^{4}$ The diagnosis of IBD is becoming more common in other races and ethnicities. A previous systematic review highlighted temporal trends in the incidence of IBD in Hispanics and Asians. ${ }^{5}$ A study from Puerto Rico reported that the crude incidence of IBD in Hispanics increased from 2.6 cases per 100,000 person-years in 1996 to 7.5 per 100,000 in $2000 .{ }^{6}$
Ther Adv Gastroenterol

2019, Vol. 12: 1-8

DOI: $10.1177 /$

1756284819827692

(c) The Author(s), 2019 Article reuse guidelines: sagepub.com/journalspermissions
Correspondence to: Edward V. Loftus, Jr Division of

Gastroenterology and Hepatology, Mayo Clinic, 200 First Street SW, Rochester MN 55905, USA Loftus.Edwardamayo.edu

Satimai Aniwan Division of

Gastroenterology and Hepatology, Mayo Clinic, Rochester, Minnesota, USA;

Division of

Gastroenterology, Chulalongkorn University, King Chulalongkorn Memorial, Hospital, Thai Red Cross Society, Bangkok, Thailand

W. Scott Harmsen Division of Biomedical Statistics and Informatics, Mayo Clinic, Rochester, Minnesota, USA

William J. Tremaine Division of

Gastroenterology and Hepatology, Mayo Clinic, Rochester, Minnesota, USA 
A study from South Korea ${ }^{7}$ showed the crude incidence of CD increased from 0.05 cases per 100,000 person-years between 1986 and 1990 to 1.34 per 100,000 person-years between 2001 and 2005, while the incidence of UC increased from 0.34 per 100,000 between 1986 and 1990 to 3.08 per 100,000 between 2001 and 2005. ${ }^{7}$ Between 2011 and 2013, an epidemiological study across eight Asian countries illustrated the pooled incidence of IBD was 1.4 cases per 100,000 personyears. ${ }^{8}$ India had the highest incidence of IBD at 9.3 cases per 100,000 person-years. ${ }^{8}$

According to the US Census Bureau in 2015, by 2051, the US population is projected to reach 400 million and to be more racially and ethnically diverse. $^{9}$ By 2044, more than $50 \%$ of the total population is projected to be belong to a 'minority group' (defined as any racial and ethnic groups other than non-Hispanic white alone).${ }^{9}$ It is therefore important to understand the burden of IBD among racial and ethnic groups in this country. Although the variation in the epidemiology of IBD among different races and ethnicities has been studied between geographic regions, population-based epidemiological data on the incidence of IBD by race and ethnicity in the US are lacking. We sought to evaluate the incidence of IBD and temporal trends from 1970 through 2010 in different racial and ethnic groups in a population-based cohort in a well-defined geographic region from Olmsted County, MN, USA.

\section{Methods}

\section{Study setting}

Olmsted County, situated in southeastern Minnesota, USA, had approximately 144,000 inhabitants at the 2010 US Census. The age, sex and ethnicity of county residents were similar to those of the state of Minnesota and the Upper Midwest. Olmsted County had less ethnic diversity than the US population (90\% versus $75 \%$ white) between 1970 and 2000. ${ }^{10}$ However, by 2010 , the racial diversity of county residents had changed to $85.7 \%$ whites, $5.4 \%$ Asians and $4.8 \%$ African Americans. There were 95.8\% non-Hispanic and $4.2 \%$ Hispanic ethnicities. ${ }^{11}$

\section{Rochester Epidemiology Project}

The resources of Rochester Epidemiology Project (REP) were used to identify permanent residents of Olmsted County, MN, who were diagnosed with IBD between 1970 and $2010 .{ }^{4}$ The REP is a unique medical records linkage system that covers the entire population of Olmsted County. It has maintained a comprehensive medical records linkage system for half a century and has been continuously funded by the National Institutes of Health. ${ }^{12}$ All of the health care for the residents of Olmsted County is provided by two organizations: Mayo Medical Center and Olmsted Medical Center. In any 4-year period, over 95\% of county residents are examined at either one of the two health care systems. ${ }^{13}$ Approximately $98 \%$ of patients provided research authorization to REP health care providers. ${ }^{13}$ Demographic data, diagnosis, surgical procedure, laboratory data and drug prescriptions are electronically recorded in indexes that can be searched by computer. Thus, this population-based data resource ensures virtually complete case ascertainment and follow up in a well-defined geographic region.

\section{Case ascertainment}

The institutional review boards of both Mayo Clinic and Olmsted Medical Center approved the study. We identified all Olmsted County residents who were first diagnosed with IBD between 1 January 1970 and 31 December 2010 according to well-defined (either CD or UC) criteria as previously described.4,14-16 Diagnosis of CD was defined as meeting at least two of the following criteria, on two occasions, separated by $\geqslant 2$ months: (1) clinical history of abdominal pain, diarrhea, weight loss, malaise, or rectal bleeding; (2) endoscopic findings of linear ulceration, mucosal cobblestoning, skip areas, or perianal disease; (3) radiologic findings of fistula, stricture, mucosal cobblestoning, or ulceration; (4) laparotomy appearance of 'creeping fat', bowel wall induration, and mesenteric lymphadenopathy; or (5) histologic findings of transmural inflammation or epithelioid granulomas. Diagnosis of UC was defined as meeting the following criteria, on two occasions, separated by $\geqslant 6$ months: diffusely granular or friable colonic mucosa; and continuous mucosal involvement based on endoscopy or barium study. ${ }^{4,14-16}$

The medical records of only patients who did not withdraw research authorization were reviewed. Demographic data including date of birth, date of IBD diagnosis, sex, race, and ethnicity were recorded. Race was categorized into white and 
nonwhite (African American, Asian, American Indian, or other/mixed). Ethnicity was categorized into Hispanic and non-Hispanic. Racial data were available from 1970 through 2010 and ethnic data were available from 1985 through 2010 .

\section{Statistical analysis}

Incidence rates in different race and ethnicity were estimated by dividing the number of county residents diagnosed with IBD by the total of population at risk. Rates were adjusted for age and sex to the 2010 US population. We also calculated the incidence rates in different race and ethnicity stratifying by calendar years of IBD diagnosis (1970-1984, 1985-1999, and 20002010). Poisson regression analysis was used to determine the association of race, ethnicity, age, sex, and calendar year of diagnosis with the incidence rates. An alpha-level of 0.05 was considered statistically significant.

\section{Results}

A total of 895 Olmsted County residents were diagnosed with IBD during the 1970-2010 period. A total of 77 patients with unknown racial and ethnic status and 4 patients who refused to provide the racial and ethnic information were excluded, leaving 814 patients in the study analysis.

\section{Incidence by race}

In the 1970-2010 period, a total of 776 whites and 38 nonwhites (13 African American, 11 Asian, 2 American Indian, 12 other/mixed) were diagnosed with IBD. The overall age- and sexadjusted incidence rates for IBD in whites and nonwhites were 21.6 cases per 100,000 personyears [95\% confidence interval (CI), 20.0-23.1] and 13 per 100,000 (95\% CI, 8.3-17.5), respectively (Table 1 ). The adjusted incidence rates for IBD increased during the 1970-2010 period in both whites and nonwhites. For whites, the adjusted IBD incidence rate was 18 cases per 100,000 person-years between 1970 and 1984, 21 per 100,000 between 1985 and 1999 and 25 per 100,000 between 2000 and 2010. For nonwhites, the adjusted IBD incidence rate was 6.4 cases per 100,000 person-years between 1970 and 1984, 9.3 per 100,000 between 1985 and 1999, and 15 per 100,000 between 2000 and
2010 (Figure 1). The 30-to-59-year-old age group had the highest crude incidence rate of IBD in both whites (24.4 cases per 1000,000 person-years) and nonwhites (19.6 per 100,000). Overall age-adjusted IBD incidence rates for males were relatively higher than those for females in both whites and nonwhites. In Poisson regression analysis, white race $(p<0.001)$, younger age $(p=0.009)$, male sex $(p<0.001)$ and a later calendar year of diagnosis $(p<0.001)$ were significantly associated with a higher incidence rate of IBD.

\section{Incidence by ethnicity}

In the 1985-2010 period, there were a total of 16 Hispanics and 623 non-Hispanics diagnosed with IBD. The overall age- and sex-adjusted incidence rates for IBD in Hispanics and non-Hispanics were 15 cases per 100,000 person-years $(95 \%$ CI, 6.323.6) and 20 per 100,000 (95\% CI, 18.5-21.6), respectively (Table 2). The adjusted incidence rate of IBD for Hispanics decreased from 25.4 per 100,000 between 1985 and 1999 to 11.1 per 100,000 between 2000 and 2010, while the rate for non-Hispanics increased from 17.4 per 100,000 between 1985 and 1999 to 23.1 per 100,000 between 2000 and 2010 (Figure 2). The ageadjusted incidence rate for females was numerically higher than males for Hispanics which was in contrast to non-Hispanics. Overall incidence rates for IBD in Hispanics were likely similar in all age groups (range, $12.3-17$ cases per 100,000 personyears). For non-Hispanics, the 30-to-59-year-old age group had the highest crude incidence rate of IBD (23 cases per 1000,000 person-years). In Poisson regression analysis, younger age $(p<0.001)$, male sex $(p=0.001)$ and a later calendar year of diagnosis $(p<0.001)$ were significantly associated with a higher incidence rate of IBD. Although nonHispanics had a higher incidence rate of IBD than Hispanics, the association between ethnicity and higher incidence rate was not statistically significant $(p=0.09)$.

\section{Discussion}

This descriptive epidemiologic study of IBD in a population-based cohort from Olmsted County, MN, USA between 1970 and 2010 demonstrated an increase in IBD incidence by race and ethnicity. Whereas the incidence of IBD in whites was 25 cases per 100,000 person-years and that of nonwhites was 15 per 100,000 in 2000-2010, the 
Table 1. Age- and sex-adjusted incidence rate for IBD by race in a population-based inception cohort from 1970 through 2010.

\begin{tabular}{|c|c|c|c|c|}
\hline \multirow[t]{2}{*}{ Race } & \multicolumn{2}{|l|}{ White } & \multicolumn{2}{|l|}{ Nonwhite } \\
\hline & IBD, $n$ & IBD incidence* & IBD, $n$ & IBD incidence* \\
\hline Overall & 776 (424 UC/352 CD) & 21.6 & 38 (19 UC/19 CD) & 13 \\
\hline \multicolumn{5}{|c|}{ By year of diagnosis } \\
\hline $1970-1984$ & 173 & 18.0 & 2 & 6.4 \\
\hline 1985-1999 & 283 & 21.0 & 5 & 9.3 \\
\hline $2000-2010$ & 320 & 25.0 & 31 & 15.0 \\
\hline \multicolumn{5}{|l|}{ By sex } \\
\hline Female & 366 & 18.9 & 14 & 10.6 \\
\hline Male & 410 & 24.4 & 24 & 14.8 \\
\hline \multicolumn{5}{|l|}{ By age, years } \\
\hline $0-29$ & 318 & 20.3 & 14 & 7.1 \\
\hline $30-59$ & 355 & 24.4 & 22 & 19.6 \\
\hline $60-99$ & 103 & 17.5 & 2 & 11.2 \\
\hline
\end{tabular}

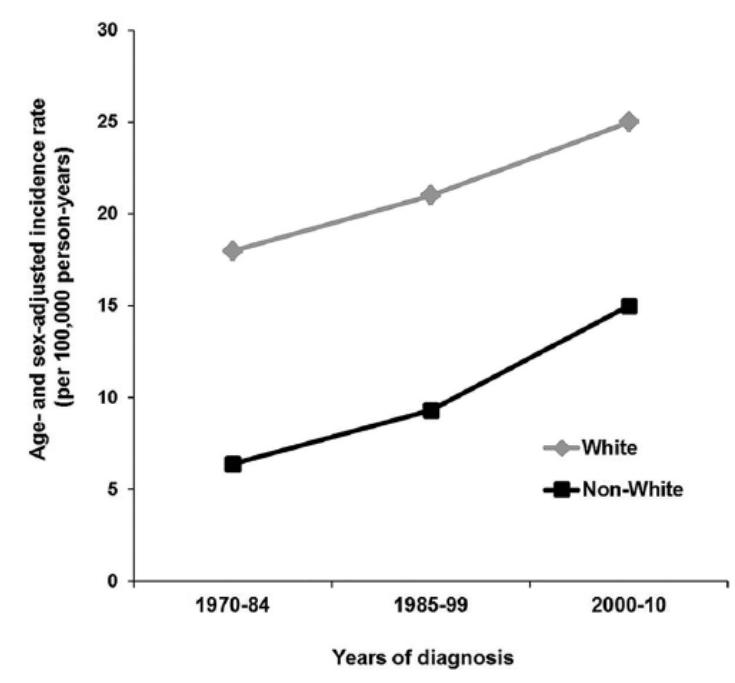

Figure 1. Age- and sex-adjusted incidence rate for IBD by race in a population-based inception cohort from 1970 through 2010.

IBD, inflammatory bowel disease.

rate had increased by $39 \%$ for whites and had sharply increased by $134 \%$ for nonwhites from 1970 through 2010. While the incidence of IBD in Hispanics was 11.1 cases per 100,000 personyears between 2000 and 2010, which had decreased by $56 \%$ from 1985 through 2010 , the incidence of IBD in non-Hispanics was 23.1 per 100,000 between 2000 and 2010, a 33\% increase from 1985 through 2010 . We found that white race, younger age, male sex and a later calendar year of diagnosis were associated with a higher incidence rate of IBD.

The racial and ethnic makeup of the USA has changed significantly in recent decades. The nonHispanic white population has decreased from $69.1 \%$ in 2000 to $62.3 \%$ in 2015 , while the Hispanic or Latino population has increased from $12.5 \%$ to $17.1 \%$ and the Asian population has increased from $3.6 \%$ to $5.1 \%$ over the same time period. ${ }^{17}$ There has been little recent research to assess the epidemiology of IBD in different racial and ethnic groups within the same geographic region. In the 1980s, a study from southern California, USA using the Kaiser Permanente Medical Care Program reported that African Americans had a CD prevalence approximately two-thirds of that of whites (29.8 cases per 
Table 2. Age- and sex-adjusted incidence rate for IBD by ethnicity in a population-based inception cohort from 1985 through 2010.

\begin{tabular}{|c|c|c|c|c|}
\hline \multirow[t]{2}{*}{ Ethnicity } & \multicolumn{2}{|l|}{ Hispanic } & \multicolumn{2}{|l|}{ Non-Hispanic } \\
\hline & IBD, $n$ & IBD incidence* & IBD, $n$ & IBD incidence* \\
\hline Overall & 16 (11 UC/5 CD) & 15 & 623 (336 UC/287 CD) & 20 \\
\hline \multicolumn{5}{|c|}{ By year of diagnosis } \\
\hline 1985-1999 & 6 & 25.4 & 282 & 17.4 \\
\hline $2000-2010$ & 10 & 11.1 & 341 & 23.1 \\
\hline \multicolumn{5}{|l|}{ By sex } \\
\hline Female & 9 & 19.8 & 286 & 17.4 \\
\hline Male & 7 & 9.4 & 337 & 23.0 \\
\hline \multicolumn{5}{|l|}{ By age, years } \\
\hline $0-29$ & 8 & 12.3 & 244 & 17.0 \\
\hline $30-59$ & 7 & 16.1 & 290 & 23.0 \\
\hline $60-99$ & 1 & 17.0 & 89 & 19.7 \\
\hline $\begin{array}{l}{ }^{*} \text { Cases per } 100 \\
\text { CD, Crohn's dis }\end{array}$ & $\begin{array}{l}\text { years. } \\
\text { flammatory bowel }\end{array}$ & ease; UC, ulcerativ & litis. & \\
\hline
\end{tabular}

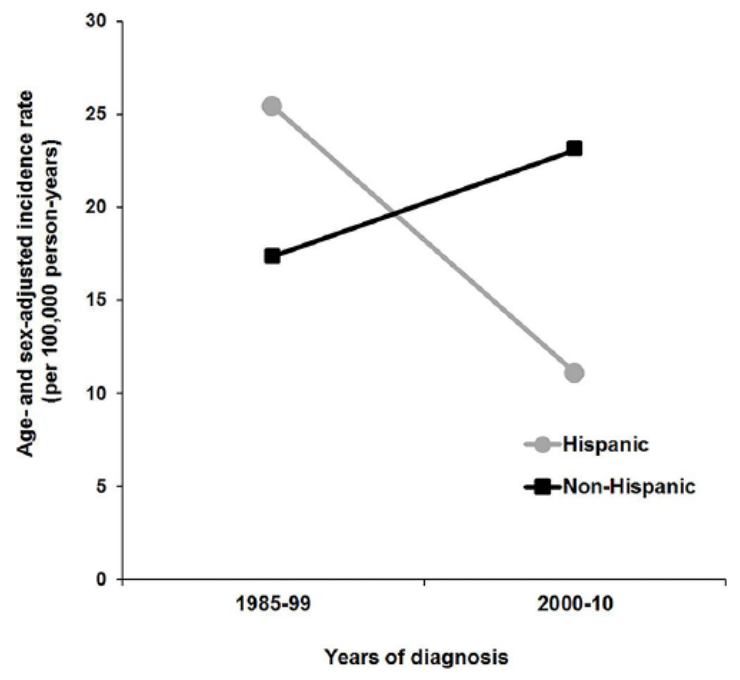

Figure 2. Age- and sex-adjusted incidence rate for IBD by ethnicity in a population-based inception cohot from 1985 through 2010.

IBD, inflammatory bowel disease.

100,000 person-years versus 43.6 per 100,000, respectively), and that Hispanics had much lower prevalence of CD (4 per 100,000). ${ }^{18}$ Nguyen and colleagues used data from the National Health Interview Study (NHIS) in 1999 to calculate the incidence of IBD among racial and ethnic groups in the US. ${ }^{19}$ They demonstrated that the estimated incidence of IBD among non-Hispanic whites was 70.2 cases per 100,000 person-years, 24.9 per 100,000 for African Americans and 9.9 per 100,000 for Hispanics. ${ }^{19}$ In contrast to our study, although the NHIS study utilized a large database, the case ascertainment of IBD was by self-report. IBD diagnosis was not verified, and it is possible that participants may have mistakenly over-reported. For example, IBD could easily be mistaken for irritable bowel syndrome. This may explain the much higher incidence of IBD relative to other reports. ${ }^{14}$

Wang and colleagues used another survey of US households (Medical Expenditure Panel Survey, 1996-2007) to estimate the prevalence of IBD by different races and ethnicities. ${ }^{20}$ Similarly, the authors showed that whites had the highest prevalence of IBD (243 cases per 100,000 personyears). The prevalence of IBD among minority groups was approximately $20-40 \%$ of that of whites. The prevalence of IBD was 96 cases per 100,000 person-years in African Americans, 85 per 100,000 in Asians, and 50 per 100,000 in Hispanics. ${ }^{20} \mathrm{~A}$ US study of the military health care 
population showed that the prevalence of IBD in whites was 324 cases per 100,000 person-years. The prevalence of IBD among minorities was almost $50-75 \%$ of that of whites: 239 per 100,000 in African Americans, 162 per 100,000 in Asians, and 147 per 100,000 in Hispanics. ${ }^{21}$ When the Medical Expenditure Panel Survey study and US military population-based study were taken into account, a trend in the increased prevalence of IBD among minorities and that of whites was observed. In our study, we found rising incidence rates of IBD in both whites (increased by 39\%) and nonwhites (increased by 134\%). The incidence rate of IBD in nonwhites between 1970 and 1984 was $35 \%$ of the rates of whites, and this percentage increased to $44 \%$ of the white incidence rate between 1985 and 1999 and to $60 \%$ between 2000 and 2010, accordingly.

It is important to understand the trend of disease subtype and characteristics among different races and ethnicities because this information may help inform the prognosis and management of IBD. Although several studies have assessed the differences in incidence of IBD subtype and disease phenotype among different racial/ethnic migrants worldwide, the findings should be interpreted with caution. A meta-analysis of CD incidence in South Asian (South East Asia and China) immigrants to the United Kingdom and Canada showed there was a trend towards lower incidence of CD [rate ratio (RR), 0.78 ; 95\% CI, 0.22-2.78] but a trend towards higher incidence of UC (RR, 1.39; 0.84-2.32) among South Asians compared with whites, while data on other racial/ethnicity groups were too limited to perform analysis. ${ }^{22}$ The phenotype of colonic CD and extensive UC was more likely to be found in South Asians than in whites. The authors also noted that the significant weaknesses of most studies included their hospital-based nature and lack of standard diagnostic criteria and disease classification. ${ }^{22}$ Another systematic review of disease characteristics of IBD among racial/ethnic minorities in the US showed no differences in disease location and behavior among those groups; however, there were only a few studies that reported disease phenotype, and the results were heterogeneous. ${ }^{23}$

Although previous studies reported the prevalence of IBD among racial and ethnic groups from the US, one must be cautious in comparing results due to different study populations and study designs. Despite large sample sizes, some administrative studies that rely on diagnostic codes have limitations, in particular misclassification. Our study differs from previous studies in that we assembled a longitudinal populationbased inception cohort within a well-defined geographic region from 1970 through 2010. All case ascertainment was based on clinical diagnostic criteria. Race and ethnic information was obtained from the electronic medical record. Each potential IBD case was manually reviewed to verify a diagnosis of IBD, race and ethnic information rather than relying on questionnaire survey or administrative data. Furthermore, our study population consisted only of permanent Olmsted County residents without influence of prevalence cases on our findings. This allowed us to determine the age- and sex-adjusted annual incidence of IBD by race and ethnicity and to also explore temporal trends. It is unclear whether differences in health-seeking behavior could change the incidence of IBD in minorities. Because of the REP, all health care in the entire Olmsted County resident population was able to be captured virtually regardless of race/ethnicity, socioeconomic status and health insurance status. ${ }^{10}$ However, there are some limitations of this study. First, the population of Olmsted County is relatively small and not as diverse racially and ethnically as that of the entire US population. Generalizability of these data to more racially and ethnically diverse populations may not be possible. Second, data for Hispanic ethnicity was only available after 1985. The prevalence of Hispanic people living in Olmsted County in 2000 was $2.4 \%$ of the total population, and the relatively rapid increase to $4.2 \%$ in 2010 may have contributed to the significant change of incidence of IBD in Hispanic people. ${ }^{11}$ However, the number of Hispanic residents may be too small to draw a definitive conclusion regarding the temporal trends. Third, we initially attempted to assess the incidence of IBD subtypes (CD and $\mathrm{UC}$ ) and disease phenotype but due to small numbers we did not perform a separate analysis by subtype. There was a relatively small number of incident CD and UC cases among different ethnic/racial groups in this study, and no conclusions could be drawn. Fourth, data on age- and sex-specificity of each race and ethnicity in the US population are not available. Therefore, we used the entire US population as a denominator for standardized calculating the ageand sex-adjusted incidence rate. 
The reasons for increasing incidence rates of IBD and significant differences by race and ethnicity are relatively unknown. It has been proposed that there are genetic, host immune response, and environmental factors that contribute to the expression of IBD. To date, more than 160 IBDsusceptible genetic loci have been identified by using genome-wide association studies. ${ }^{24}$ Recent studies and a meta-analysis showed that many of the IBD risk loci are shared among different racial and ethnic ancestries including whites, African Americans and Asians. ${ }^{25,26}$ Although there has been greatly increased understanding of the genetic risk for IBD, it remains unclear why most individuals who carry the risk loci remain healthy whereas others develop IBD. Emerging evidence suggests that environmental factors and their interaction with the host immune system in the genetically susceptible individual may be the fundamental development of IBD. ${ }^{27}$ A study from Canada reported that immigrants to Ontario from all regions had a lower incidence of IBD as compared with nonimmigrants (incidence rate ratio, $0.34 ; 95 \% \mathrm{CI}$, $0.26-0.44) .{ }^{28}$ However, the Ontario-born children of mothers who were immigrants from Middle East/North Africa, South Asia, SubSahara Africa, western Europe and North America had similar relative incidence of IBD as children of nonimmigrants, while the incidence of IBD among the second generation of immigrants from some regions continued to be lower compared with children of nonimmigrants. ${ }^{28}$ Although our study was not designed to answer this question, our findings partially provide clues for further investigations regarding the relationship between the genetic susceptibility and environmental determinants of IBD development.

In summary, there were significant differences in the incidence rate and temporal trends of IBD among racial and ethnic groups over the last four decades in this population-based cohort. An increase in the incidence of IBD for whites and nonwhites leads to an increased health economic burden in the future. Extrapolating the adjusted annual incidence to the 2010 US census, there are approximately 55,000 white and 11,000 nonwhite US residents newly diagnosed with IBD annually, for a total of about 66,000 US residents with a new IBD diagnosis each year. However, these estimated numbers should be considered a rough approximation of the true incident IBD cases in the US, our population are fairly heterogenous in race and ethnicity as compared with the entire US population. To prepare for the changing landscape of the burden of IBD over the next decade with more racial and ethnic diversity, further research is warranted to understand the etiology of these differences.

\section{Acknowledgements}

The authors are grateful to Lawrence Timmons and Debra Jewell for data abstraction.

\section{Funding}

This work was supported in part by the Mayo Foundation for Medical Education \& Research, and the REP (grant number R01 AG034676 from the National Institute on Aging of the National Institutes of Health). The contents of the publication are solely the responsibility of the authors and do not necessarily represent the official view of the National Institutes of Health.

\section{Conflict of interest statement}

The authors declare that there is no conflict of interest.

\section{ORCID iD}

Satimai Aniwan (D) https://orcid.org/0000-0002 $-2730-2450$

\section{References}

1. Jess T, Loftus EV Jr, Harmsen WS, et al. Survival and cause specific mortality in patients with inflammatory bowel disease: a long-term outcome study in Olmsted County, Minnesota, 19402004. Gut 2006; 55: 1248-1254.

2. Molodecky NA, Soon IS, Rabi DM, et al. Increasing incidence and prevalence of the inflammatory bowel diseases with time, based on systematic review. Gastroenterology 2012; 142: 46-54.e42; quiz e30.

3. Kaplan GG. The global burden of IBD: from 2015 to 2025. Nat Rev Gastroenterol Hepatol 2015; 12: 720-727.

4. Shivashankar R, Tremaine WJ, Harmsen WS, et al. Incidence and prevalence of Crohn's disease and ulcerative colitis in Olmsted county, Minnesota from 1970 through 2010. Clin Gastroenterol Hepatol 2017; 15: 857-863.

5. Hou JK, El-Serag $\mathrm{H}$ and Thirumurthi S. Distribution and manifestations of inflammatory bowel disease in Asians, Hispanics, and African Americans: a systematic review. $A m \mathcal{F}$ Gastroenterol 2009; 104: 2100-2109. 
6. Appleyard CB, Hernandez G and Rios-Bedoya CF. Basic epidemiology of inflammatory bowel disease in Puerto Rico. Inflamm Bowel Dis 2004; 10: 106-111.

7. Yang SK, Yun S, Kim JH, et al. Epidemiology of inflammatory bowel disease in the SongpaKangdong district, Seoul, Korea, 1986-2005: a KASID study. Inflamm Bowel Dis 2008; 14: 542-549.

8. Ng SC, Tang W, Ching JY, et al. Incidence and phenotype of inflammatory bowel disease based on results from the Asia-pacific Crohn's and colitis epidemiology study. Gastroenterology 2013; 145: 158-165.e2.

9. Sandra LC and Ortman JM. Projections of the size and composition of the US population: 2014 to 2060. Washington, DC: United States Census Bureau, 2015.

10. St Sauver JL, Grossardt BR, Leibson CL, et al. Generalizability of epidemiological findings and public health decisions: an illustration from the Rochester Epidemiology Project. Mayo Clin Proc 2012; 87: 151-160.

11. United States Census Bureau. Census 2010 total population and description in Olmsted county, Minnesota, https://factfinder.census.gov/faces /nav/jsf/pages/community_facts.xhtml?src=bkmk (accessed 17 July 2017).

12. Rocca WA, Yawn BP, St Sauver JL, et al. History of the Rochester Epidemiology Project: half a century of medical records linkage in a US population. Mayo Clin Proc 2012; 87: 1202-1213.

13. St Sauver JL, Grossardt BR, Yawn BP, et al. Use of a medical records linkage system to enumerate a dynamic population over time: the Rochester epidemiology project. Am F Epidemiol 2011; 173: 1059-1068.

14. Loftus CG, Loftus EV Jr, Harmsen WS, et al. Update on the incidence and prevalence of Crohn's disease and ulcerative colitis in Olmsted County, Minnesota, 1940-2000. Inflamm Bowel Dis 2007; 13: 254-261.

15. Loftus EV Jr, Silverstein MD, Sandborn WJ, et al. Crohn's disease in Olmsted County, Minnesota, 1940-1993: incidence, prevalence, and survival. Gastroenterology 1998; 114: 1161-1168.

16. Loftus EV Jr, Silverstein MD, Sandborn WJ, et al. Ulcerative colitis in Olmsted County, Minnesota, 1940-1993: incidence, prevalence, and survival. Gut 2000; 46: 336-343.

Visit SAGE journals onlin journals.sagepub.com/ home/tag

(SAGE journals .census.gov/prod/cen2010/briefs/c2010br-02.pdf (accessed 15 October 2018).

18. Kurata JH, Kantor-Fish S, Frankl H, et al. Crohn's disease among ethnic groups in a large health maintenance organization. Gastroenterology 1992; 102: 1940-1948.

19. Nguyen GC, Chong CA and Chong RY. National estimates of the burden of inflammatory bowel disease among racial and ethnic groups in the United States. F Crohns Colitis 2014; 8: 288-295.

20. Wang YR, Loftus EV Jr, Cangemi JR, et al. Racial/ethnic and regional differences in the prevalence of inflammatory bowel disease in the United States. Digestion 2013; 88: 20-25.

21. Betteridge JD, Armbruster SP, Maydonovitch C, et al. Inflammatory bowel disease prevalence by age, gender, race, and geographic location in the U.S. military health care population. Inflamm Bowel Dis 2013; 19: 1421-1427.

22. Misra R, Faiz O, Munkholm P, et al. Epidemiology of inflammatory bowel disease in racial and ethnic migrant groups. World $\mathcal{F}$ Gastroenterol 2018; 24: 424-437.

23. Afzali A and Cross RK. Racial and ethnic minorities with inflammatory bowel disease in the United States: a systematic review of disease characteristics and differences. Inflamm Bowel Dis 2016; 22: 2023-2040.

24. Jostins L, Ripke S, Weersma RK, et al. Hostmicrobe interactions have shaped the genetic architecture of inflammatory bowel disease. Nature 2012; 491: 119-124.

25. Franke A, McGovern DP, Barrett JC, et al. Genome-wide meta-analysis increases to 71 the number of confirmed Crohn's disease susceptibility loci. Nat Genet 2010; 42: 1118-1125.

26. Liu JZ, van Sommeren S, Huang H, et al. Association analyses identify 38 susceptibility loci for inflammatory bowel disease and highlight shared genetic risk across populations. Nat Genet 2015; 47: 979-986.

27. Sheehan D and Shanahan F. The gut microbiota in inflammatory bowel disease. Gastroenterol Clin North Am 2017; 46: 143-154.

28. Benchimol EI, Mack DR, Guttmann A, et al. Inflammatory bowel disease in immigrants to Canada and their children: a population-based cohort study. Am $\mathcal{F}$ Gastroenterol 2015; 110 : 553-563. 